\title{
Erratum: Time-dependent approach to electron transport through junctions: General theory and simple applications [Phys. Rev. B 22, 5887 (1980)]
}

\author{
Michele Cini* \\ (Received 9 May 2014; published 9 June 2014)
}

DOI: 10.1103/PhysRevB.89.239902

PACS number(s): 73.40.Gk, 99.10.Cd

In Eq. (59b) several "2" should be replaced by " $r$ ". The equation should read

$$
J(V)=-\frac{4 e \epsilon_{F}}{\pi \hbar} \int_{1}^{1+r} d x \Theta(x-r) \frac{\sqrt{x(x-r)}}{|\sqrt{x}+\sqrt{x-r}|^{2}} .
$$

There is also a typo in Eq. (85). It should read

$$
J(V)=\frac{8 e T}{N \hbar} \sum_{q} f_{q} \sin ^{2}(q a)\left(\frac{\Theta(q) \operatorname{Im} A\left(\epsilon_{q}+V\right)}{\left|A\left(\epsilon_{q}+V\right)-e^{-i q a}\right|^{2}}-\frac{\Theta(-q) \operatorname{Im} B\left(\epsilon_{q}\right)}{\left|B\left(\epsilon_{q}\right)-e^{-i q a}\right|^{2}}\right) .
$$

In particular, at half filling $\left(q_{F} a=\frac{\pi}{2}\right)$ and zero temperature, by changing the integration variable from $q$ to $\cos (q a)$ and doing some algebra, this leads to Eq. (86).

These are typing errors that escaped my attention when reading the proofs and do not affect any of the results and conclusions of this paper.

*Present address: Dipartimento di Fisica, Università di Roma Tor Vergata, via della Ricerca Scientifica 1, I-00133 Roma, Italy and INFN, Laboratori Nazionali di Frascati, via E. Fermi 40, 00044 Frascati, Italy. 